\section{ADENOVIRUSES \\ Ad2 and SV40}

from our Cell Biology Correspondent WHAT renders a cell non-permissive and unable to support the replication of any particular virus is one of the more interesting questions which virologists face and no doubt the molecular basis of non-permissiveness will prove to be manifold, with different mechanisms operating against different viruses in different cells.

One of the few pertinent cell-virus combinations that has been investigated in some detail is the infection of African green monkey kidney (AGMK) cells by human adenovirus type 2 (Ad2). This virus cannot replicate efficiently in AGMK cells even though the virion penetrates the cells, is uncoated and its DNA is transcribed and replicated. When, however, Ad2 and simian virus 40 coinfect AGMK cells the yield of progeny $\mathrm{Ad} 2$ is increased by about 1,000-fold. Precisely how SV40 acts as a helper for the replication of $\mathrm{Ad} 2$ in these cells and renders them permissive remains unknown, but recent experiments carried out by Lucas and Ginsberg (J. Virology, 10, 1109; 1972) seem to eliminate all but two possibilities; namely either SV40 protects some Ad2 mRNAs from partial degradation by endonucleases or SV40 specifies some function which allows late Ad2 messengers to be translated.

Lucas and Ginsberg have used DNARNA hybridization techniques to compare the extent of transcription of the Ad2 genome in AGMK cells, in AGMK cells coinfected with SV40 and in permissive human $\mathrm{KB}$ cells in which the adenovirus replicates without the aid of SV40. They also used immunological precipitation techniques to compare the amounts of adenovirus structural proteins made in these various infections. The hybridization experiments, which were carefully conducted to eliminate artefacts, reveal that both early and late species of Ad2 messengers are synthesized in KB cells and AGMK cells in the absence of SV40. Furthermore, the non-conservative maturation of these messengers and their transport to the cytoplasm are comparable in both sorts of cells. In AGMK cells, however, in the absence of SV40 the synthesis of adenovirus structural proteins is some 90-fold less than the synthesis achieved in the presence of SV40 helper. Apparently therefore AGMK cells support transcription of Ad2 DNA, but the viral RNA is not translated.

Lucas and Ginsberg do not yet know whether or not the late Ad2 messengers are partially degraded and not available for translation. If it proves that these RNAs are degraded, and they are addressing themselves to this question,
SV40 must somehow protect the molecules from the endonuclease(s). If, on the other hand, the late Ad2 messengers in AGMK cells are as stable as they are in KB cells Lucas and Ginsberg will look for a function specified by SV40 that directly promotes the translation of Ad2 messengers. The result, whatever it is, will be interesting.

In the same issue of the journal (ibid., 1126) Bhadani, Raskas and Green report a method for partially purifying milligram amounts of Ad2 messenger RNA from KB cells some 18 hours after infection. They have simply exploited the fact that at high ionic strengths the polyadenylic acid tails of the messengers cause them to bind to cellulose nitrate filters. As they point out, these mRNAs should prove useful for analysing the synthesis of adenovirus proteins in cell-free systems and, of course, if Lucas and Ginsberg find that the SV40 helper function is directly involved in facilitating translation, such cell-free systems may provide a means of identifying precisely the helper function.

\section{DATING TECHNIQUES \\ Using Carbon-14}

from a Correspondent

THE intention of the eighth international conference on radiocarbon dating, which was organized by the Royal Society of New Zealand at Lower Hutt on October 18-25, was to bring together scientists engaged in operating dating laboratories and in evaluating results. The programme was designed to deal with technical aspects of dating and the significance of isotopic ratios measured in the estimation of radiocarbon dates and with the environmental factors that might affect the interpretation of the results in terms of absolute years. It was not intended to provide a forum for the discussion of actual projects using carbon-14 dates primarily as indicators of time supporting other geochemical or archaeological evidence. The chief emphasis was on the problems of the variation of concentration and residence times of carbon-14 in ocean water; the role of radiocarbon in freshwater and in soil development the effects and amounts of various contaminants on dates; and on considerations of suitable standards for carbon-14 activity.

It was appropriate that the meeting was held at Lower Hutt-nine miles from Wellington-because this is the site of the New Zealand Department of Scientific and Industrial Research whose director, Dr A. Rafter, with the collaboration of $\mathrm{Dr}$ G. J. Fergusson, established the first carbon-14 dating laboratory, with a range surpassing 55,000 years, in the Southern Hemisphere.

Of general interest were the contributions on secular variations of atmospheric radiocarbon. There are three distinct areas. Dr W. Fergusson (University of Arizona) reported that the bristlecone pine chronology had been extended by another 1,000 years to nearly 8,200 BP and that he would shortly have wood of this age available for radiocarbon analysis. Dr E. Ralph (University of Pennsylvania) and Professor P. Damon (University of Arizona) described separate methods for dealing with the nearly 400 determinations in the literature of the radiocarbon ages of dendrochronologically

\title{
Inducing Polyoma Virus Production
}

IN Nature New Biology next Wednesday (February 7) Fogel describes further experiments with the line of rat embryo muscle cells transformed by the large plaque strain of polyoma virus which he and Sachs established in 1967. This line of transformed cells is of interest because at a frequency of about 1 in $10^{4}$ the cells are spontaneously induced to release polyoma virus. Furthermore, various physical and chemical agents which cause DNA breakage increase the frequency of induction of virus synthesis.

In his latest experiments Fogel allowed the cells to incorporate varying amounts of 5-bromodeoxyuridine and then exposed them to various doses of visible light, a procedure which results in single strand breaks in the cell DNA. $\mathrm{He}$ reports that after such treatment the number of cells containing polyoma virus capsid antigen can be increased by some 600 -fold and the number of cells producing virus can be increased by about 100 -fold. The number of infectious virus particles produced per cell, however, decreases.

One obvious explanation of these data is that the extensive DNA breakage induced by the treatment is lethal to the cells which do not survive long enough to produce much virus. This idea is supported by the observation that exposure of the cells to cysteamine at the time of irradiation increases cell survival up to 70-fold, decreases some 2-3-fold the number of cells containing capsid antigen but increases the number of virus particles produced per cell. AIl these data indicate that the replication of polyoma virus in these cells is induced by single strand breakage of the cellular DNA. 